\title{
Qualitative study exploring the barriers to menstrual hygiene management faced by adolescents and young people with a disability, and their carers in the Kavrepalanchok district, Nepal
}

Jane Wilbur $^{1 *} \mathbb{B}$, Shubha Kayastha² ${ }^{2}$ Thérèse Mahon ${ }^{3}$, Belen Torondel ${ }^{1}$, Shaffa Hameed ${ }^{1}$, Anita Sigdel², Amrita Gyawali ${ }^{2}$ and Hannah Kuper ${ }^{1}$

\begin{abstract}
Background: Menstrual hygiene management (MHM) is a recognised public health, social and educational issue, which must be achieved to allow the realisation of human rights. People with disabilities are likely to experience layers of discrimination when they are menstruating, but little evidence exists.

Methods: The study aims to investigate barriers to MHM that people with disabilities and their carers face in the Kavrepalanchok, Nepal, using qualitative methods. Twenty people with disabilities, aged 15-24, who menstruate and experience 'a lot of difficulty' or more across one or more of the Washington Group functional domains were included, as well as 13 carers who provide menstrual support to these individuals. Purposeful sampling was applied to select participants. Different approaches were used to investigating barriers to MHM and triangulate data: indepth interviews, observation, PhotoVoice and ranking. We analysed data thematically, using Nvivo 11.

Results: Barriers to MHM experienced by people with disabilities differ according to the impairment. Inaccessible WASH facilities were a major challenge for people with mobility, self-care and visual impairments. People with intellectual impairments had difficulty accessing MHM information and their carers despaired when they showed their menstrual blood to others, which could result in abuse. No support mechanisms existed for carers for MHM, and they felt overwhelmed and isolated. Menstrual discomfort was a major challenge; these were managed with home remedies, or not at all. Most participants followed menstrual restrictions, which were widespread and expected; many feared they would be cursed if they did not. As disability is often viewed as a curse, this demonstrates the layers of discrimination faced.

(Continued on next page)
\end{abstract}

\footnotetext{
* Correspondence: jane.wilbur@lshtm.ac.uk

'London School of Hygiene \& Tropical Medicine, Keppel Street, London

WC1E 7HT, UK

Full list of author information is available at the end of the article
}

C C The Author(s). 2021 Open Access This article is licensed under a Creative Commons Attribution 4.0 International License, which permits use, sharing, adaptation, distribution and reproduction in any medium or format, as long as you give appropriate credit to the original author(s) and the source, provide a link to the Creative Commons licence, and indicate if changes were made. The images or other third party material in this article are included in the article's Creative Commons licence, unless indicated otherwise in a credit line to the material. If material is not included in the article's Creative Commons licence and your intended use is not permitted by statutory regulation or exceeds the permitted use, you will need to obtain permission directly from the copyright holder. To view a copy of this licence, visit http://creativecommons.org/licenses/by/4.0/ The Creative Commons Public Domain Dedication waiver (http://creativecommons.org/publicdomain/zero/1.0/) applies to the data made available in this article, unless otherwise stated in a credit line to the data. 
(Continued from previous page)

Conclusion: Issues related to MHM for people with disabilities is more complex than for others in the population due to the additional disability discrimination and impairment experienced. Research exploring these issues must be conducted in different settings, and MHM interventions, tailored for impairment type and carers requirements, should be developed. Attention to, and resourcing for disability inclusive MHM must be prioritised to ensure 'no one is left behind'.

Keywords: Disability, Carers, Menstrual hygiene management, Water, Sanitation and hygiene, Rights

\section{Background}

Menstrual hygiene management (MHM) is a recognised public health, social and educational issue [1]. Research shows that the realisation of human rights is inhibited by lack of provision for MHM, including the right to education, health and work [2-7]. This can happen when there is: inadequate physical water, sanitation and hygiene (WASH) infrastructure to support menstruation at home and in public spaces, a lack of affordable, comfortable and appropriate menstrual products, a lack of accurate information on the menstrual cycle and how to manage it with dignity, as well as harmful social beliefs and taboos related to menstruation.

Underlying these issues is menstrual stigma, which is rooted in power and gender inequalities, and means that menstruation is not often openly discussed. This discourages sharing accurate information on the menstrual cycle, and how to manage it hygienically and with dignity [8]. It also leads people to be unsure how they can seek support at home, at school or through healthcare services [8]. This is the case in Nepal, where menstruation is not spoken about openly, and many prepubescent girls do not receive information about menstruation, so their first menstrual cycle can be a frightening experience $[9,10]$. Accurate information on the menstrual cycle and how to manage it hygienically is likewise inadequate [11]. Management of menstrual discomfort is limited and menstrual hygiene information is predominantly shared between family members, and focuses on the use of menstrual products and maintaining the current social beliefs and taboos surrounding menstruation. In this context, unhygienic practices are common [9].

Cultural, religious and behavioural expectations related to menstruation varies globally and within countries, as does the extent to which these impact on people's ability to fully participate in society when they are menstruating. A recent study in Nepal, found that $89 \%$ of women and girls experienced restrictions whilst menstruating [12], which involves the seclusion from the community and within the home $[10,13,14]$. Within Nepal, the extent to which menstrual restrictions are followed relates to caste and religion; $81.3 \%$ of the population are Hindu and adhere to the caste system, which is based on ritual impurity and purity $[11,15]$. There are four broad castes: Brahmin, Kshatriya, Vaishya, Sudra, and these can determine individual's behaviours, including how strictly menstrual restrictions are followed. For instance, Brahmins are the upper-caste, and tend to follow menstrual restrictions closely so that they are not contaminated by menstrual blood [12, 16, 17].

Evidence about how a lack of MHM negatively impacts gender equality is growing $[1,18,19]$. However, there is a consistent blind spot on disability, even though $15 \%$ of the world's population have a disability, and $80 \%$ of people with disabilities live in low- and middle-income countries (LMICs) [20]. Where studies do exist, they focus on a specific impairment group such as intellectual, visual or mobility instead of gaining a holistic view of the barriers faced by people with different impairments [21-23]. Within South Asia, only one study explores the MHM experiences of people with intellectual impairments and their carers [22]. With a dearth of evidence on the MHM requirements of people with disabilities and their carers, very few interventions exist $[5,24]$.

This lack of attention is compounded by disability discrimination, demonstrated by misconceptions. These include that people with disabilities do not have the same reproductive systems as non-disabled people, so may not menstruate and cannot have children, or people with disabilities are considered contagious, dirty and impure [20, 25-27]. Without social assistance, carers struggle to support another person's menstrual cycle [22, 28-30]. Management strategies applied include putting the person with a disability on long-term contraception, limiting their physical mobility during menstruation and sterilisation [22, 29-32]. Therefore, people with disabilities living in LMICs may face layers of discrimination when they are menstruating. These may negatively impact on the extent to which they can fulfil their human rights, including education, water and sanitation, and sexual and reproductive rights, but more evidence is required [5, 33, 34]. The study aims to begin filling this gap by investigating the barriers to MHM that adolescents and young people with a disability and their carers face in the Kavrepalanchok (Kavre) district, Nepal, using qualitative methods. 


\section{Methods}

\section{Research design}

Phenomenological research methodology underpins this study, and influenced the data collection tools applied [35]. We recognise menstruation as a physiological and social phenomenon: a participant's life experiences of menstruation are situated within socio-cultural factors, and menstrual related behaviours and opinions are shaped by individual and external influences. Our data collection methods guided participants to describe their lived experiences, beliefs and feelings about menstruation visually and verbally, and we observed the behaviour setting by carrying out accessibility and safety audits of the MHM facilities used. Interactions were reciprocal, and researchers answered participants' questions about menstruation, providing accurate information on the menstrual cycle and how to manage it hygienically.

\section{Research team and training}

Qualitative data was generated by the lead author (JW), a Nepali Research Coordinator (SK) and two Nepali Field Researchers (AS and AG). As we are committed to disability-inclusive research, we recruited AS (with a visual impairment) and AG (who has a mobility impairment) as field researchers and JW and SK mentored them throughout the data collection so that they could develop their research skills. All the research team members were women.

The research team participated in a week-long training, led by the lead author and a second experienced qualitative researcher on how to conduct research ethically with people who have a disability and their carers [36]. Participants included eight people with a range of disabilities, sign language interpreters, SK, and representatives from WaterAid, CBM and Plan Nepal. Attendees were involved in this study, and the 'Strengthening voices of adolescents with disabilities in Nepal' research, which aimed to understand what was important for the wellbeing of adolescents with disabilities [37]. The latter was conducted by the London School of Hygiene and Tropical Medicine (LSHTM), CBM and Plan International and started soon after the research training workshop. Participatory methods, including the Age Line [38], Feeling Dice [39], the Johari Window [40] and Collage [41], were applied to facilitate discussions on seeking informed consent, MHM, and confidently but sensitively discussing 'private' topics [42]. Guidance was given on how to generate data with people who have different impairments using qualitative methods, including ensuring breaks are regularly offered to participants throughout the process, always addressing the person with a disability directly and not an interpreter or carer, being encouraging, patient and respectful at all times.
Specifically, when interviewing people with a hearing impairment, ensuring sign-language interpretation is available if required, speaking slowly, clearly and at a steady rhythm and never shouting. When interviewing people with visual impairments, identifying yourself clearly and introducing everyone in the room; not leaving when the person is talking, and asking the participant if they prefer to sit in bright light or shade as this may affect their vision. Researchers should sit at the same level as a person with a mobility impairment, and not push a wheelchair without asking the person first. For people with intellectual impairments, carers should be present; researchers should always acknowledge the participant's contribution, speak in short, simple sentences and explain more than once if the person does not understand. Researchers should also be guided by the person's body language and end the interview if the participant becomes disengaged. Data collection tools used in this study were tested and refined during this training.

\section{Study site}

The Kavre district was selected as the study site as the research partner, WaterAid implements MHM programmes there with local NGOs, Karnali Integrated Rural Development and Research Centre (KIRDAC) and Centre for Centre for Integrated Urban Development (CIUD). The Kavre district is one of Nepal's 77 districts, with a population of 381,937 , it is classified as 'mid-hilly' [43]. The district's basic water coverage is $89 \%$ and basic sanitation coverage is $98 \%$ (unpublished data). The Kavre district was the epicentre of the 2015 earthquakes and much of its infrastructure was destroyed, including household latrines. Efforts to rebuild infrastructure are ongoing.

\section{Study population and sample size}

The study population and inclusion criteria comprised:

1) 20 individuals, aged between 15 and 24 years, who menstruate and experience'a lot of difficulty', or more across one or more of the visual, hearing, mobility, cognition, self-care and communicationfunctional domains [44].

2) 13 carers, who were selected if they provided menstrual care for a participant with a disability. Carers were interviewed about their experiences of supporting another person's menstrual cycle, as well as their interpretation of the person with disability's MHM experiences and related feelings.

Table 1. details the study population characteristics, including ages, locations and functional domains.

We applied purposeful sampling to select participants who experience the phenomenon researched $[45,46]$. 
Table 1 Study population characteristics

\begin{tabular}{|c|c|c|}
\hline Study population & Variables & $n=$ \\
\hline \multirow[t]{13}{*}{ Person with a disability } & Age group & \\
\hline & $15-19$ & 9 \\
\hline & $20-24$ & 11 \\
\hline & Location & \\
\hline & Urban & 16 \\
\hline & Rural & 4 \\
\hline & Functional c & \\
\hline & Visual & 3 \\
\hline & Hearing & 2 \\
\hline & Mobility & 8 \\
\hline & Cognition & 1 \\
\hline & Self-care & 1 \\
\hline & Multiple* & 5 \\
\hline \multirow[t]{11}{*}{ Carer } & Location & \\
\hline & Urban & 6 \\
\hline & Rural & 7 \\
\hline & Functional c & son with a disability \\
\hline & Visual & 1 \\
\hline & Mobility & 3 \\
\hline & Cognition & 7 \\
\hline & Multiple* & 2 \\
\hline & Person provi & \\
\hline & Relative & 11 \\
\hline & Professional & 2 \\
\hline
\end{tabular}

*Multiple included: mobility, cognition, self-care and communication

Firstly, the lead author explained the Washington Group short set of questions [25] to WaterAid's partner organisations: KIRDAC, CIUD and government social mobilisers, who had knowledge of people with disabilities living in the study area. The Washington Group Short Set, is a tool recommended for data disaggregation, and includes questions about a person's abilities across six functional domains: visual, hearing, mobility, cognition, self-care, and communication [44]. Disability is identified as anyone having at least 'a lot of difficulty' in one or more of these domains. Functional domains are referred to as 'impairments' in this article, and cognition as an 'intellectual impairment'.

These representatives identified 20 females with a disability, aged between 15 and 24. Secondly, the research team visited the potential participants and asked them the Washington Group short set of questions [25], their age and if they menstruate to confirm that they met the inclusion criteria. Participants that did not qualify were excluded. We intentionally selected people with a range of impairment types, so we applied snowball sampling to ensure representation across the six functional domains. Snowball sampling is a method of increasing the sample by asking participants to identify other people to interview $[47,48]$. Carers were selected if they provided menstrual care for a participant with a disability.

\section{Data collection methods and activities}

Data collection was carried out in September 2017. The study applied four different qualitative methods to allow for methods triangulation: In-depth interviews, observation and PhotoVoiceand ranking (described in Table 2) [50]. Additional Files 1-3 contain the in-depth interview guide for carers and people with disabilities, and PhotoVoice guidance, which were developed for, and used in this study. PhotoVoice participants were selected after their in-depth interview; they represented different impairments (mobility, self-care) and roles (person who menstruates, or supports another's menstrual cycle), settings (household and residential institution) and spoke about their menstrual experiences openly. PhotoVoice can be a very effective method for a person to represent their experiences visually, but it can be time intensive and takes approximately one day to complete. We aimed to complete PhotoVoice with four participants with a disability and two carers, but two individuals declined.

At the end of each day, the research team met to discuss findings and themes; when all topics were explored and no new data emerged, we concluded that data saturation was met and stopped data collection.

\section{Data analyses}

Data analyses was iterative: the research team met at the end of each day to review field notes, discuss their influence within the data collection process, and emerging themes (such as similarities of barriers to accessing MHM facilities for people with mobility impairments, or a lack of support for carers of persons with disabilities), as well as to review and revise the interview technique and topic guides. When data collection was complete, voice recordings of interviews were translated and transcribed verbatim into English. Transcriptions were checked for accuracy by Nepali researchers in the team and WaterAid Nepal staff. Transcripts were not returned to participants for comment or correction, but overall research findings were shared with participants at a later date (see Wilbur and Bright, 2019 [24]).

The lead author then applied a thematic analytical approach to analyse transcriptions, which involved: 1) familiarisation with the data, 2) generation of initial codes that were structured according to outcomes in the adapted socio-ecological framework for menstrual hygiene management [5]. Outcomes included: access to water and sanitation facilities, including menstrual materials disposal mechanisms, appropriate menstrual 
Table 2 Summary of methods

\begin{tabular}{|c|c|c|c|c|}
\hline Method & Purpose & Description & Sample characteristics & Sample size \\
\hline \multirow[t]{2}{*}{$\begin{array}{l}\text { In-depth } \\
\text { interview }\end{array}$} & $\begin{array}{l}\text { To understand barriers to MHM, and } \\
\text { how these effect participants' lives. }\end{array}$ & $\begin{array}{l}\text { Undertaken at the participant's home, } \\
\text { school, care home or hospital; } \\
\text { interviews lasted between } 1 \text { and } 1.5 \text { h. } \\
\text { With consent, interviews were } \\
\text { conducted in Nepali, recorded on a } \\
\text { voice recorder, and translated into } \\
\text { English if JW (who does not speak } \\
\text { Nepali) was present. Field notes were } \\
\text { made after the interviews. }\end{array}$ & $\begin{array}{l}\text { Individuals with disabilities, aged 15- } \\
24 \text { and menstruates, and carers who } \\
\text { support them }\end{array}$ & $\begin{array}{l}20 \\
\text { individuals } \\
\text { with } \\
\text { disabilities } \\
13 \text { carers }\end{array}$ \\
\hline & $\begin{array}{l}\text { To understand the menstrual products } \\
\text { available, user preference and } \\
\text { rationale. }\end{array}$ & $\begin{array}{l}\text { Market survey, product attribute } \\
\text { assessment and user preference with } \\
\text { ranking: a range of menstrual products } \\
\text { available on the local market were } \\
\text { displayed to participants during } \\
\text { interviews. Researchers asked } \\
\text { participants if they had used any, their } \\
\text { preference with reasons for why, and } \\
\text { to rank the products according to } \\
\text { preference. A photo was taken of the } \\
\text { products in ranked order. }\end{array}$ & Individuals with disabilities & $\begin{array}{l}16 \\
\text { individuals } \\
\text { with } \\
\text { disabilities }\end{array}$ \\
\hline Observation & $\begin{array}{l}\text { To observe if any participants face } \\
\text { accessibility or safety barriers when } \\
\text { using water, sanitation and menstrual } \\
\text { hygiene management facilities } \\
\text { (revised version of WEDC, WaterAid } \\
\text { (2013) Accessibility and safety audit } \\
\text { [49]). }\end{array}$ & $\begin{array}{l}\text { Observed participants demonstrating } \\
\text { where they stored their menstrual } \\
\text { product, how they changed, washed } \\
\text { and/or disposed of it; where they } \\
\text { collected water from, what soap was } \\
\text { used, and where they washed their } \\
\text { bodies. Issues explored included } \\
\text { accessibility, privacy and safety of } \\
\text { facilities, such as distance to water } \\
\text { source, ability to use facilities } \\
\text { independently, if the participant can } \\
\text { be seen in the facility; if it is well lit, if } \\
\text { it has a door with a lock that can be } \\
\text { used independently. Photographs of } \\
\text { facilities were taken and field notes } \\
\text { recorded. Observation took place after } \\
\text { in-depth interviews. }\end{array}$ & Individuals with disabilitiesand carers & $\begin{array}{l}20 \\
\text { individuals } \\
\text { with } \\
\text { disabilities } \\
13 \text { carers }\end{array}$ \\
\hline PhotoVoice & $\begin{array}{l}\text { To allow participants to express } \\
\text { themselves visually; allow participants } \\
\text { time to reflect on the issues, and rank } \\
\text { their experiences against perceived } \\
\text { levels of importance. }\end{array}$ & $\begin{array}{l}\text { Cameras were lent to participants, who } \\
\text { were asked to take five photos of their } \\
\text { own menstrual experience or of caring } \\
\text { for another person's menstrual cycle. } \\
\text { Photos were printed and discussed } \\
\text { with the participants, who provided } \\
\text { captions and ranked the photos } \\
\text { according to which was the greatest } \\
\text { to least important issue. The whole } \\
\text { process took } 0.5 \text { to } 1 \text { day per } \\
\text { participant. All participants requested } \\
\text { that their real names are credited } \\
\text { whenever their photos and captions } \\
\text { are used. Participant's names are used } \\
\text { in this article. }\end{array}$ & $\begin{array}{l}\text { Individuals with disabilities, able to } \\
\text { understand the task, use a camera or } \\
\text { direct a third party to take photos. } \\
\text { Carer who provides care throughout } \\
\text { the menstrual cycle. }\end{array}$ & $\begin{array}{l}3 \text { individuals } \\
\text { with } \\
\text { disabilities } \\
1 \text { carer }\end{array}$ \\
\hline
\end{tabular}

materials, relationship with carers / the person with a disability, ability to manage menstruation independently, ability to manage another person's menstrual cycle and tasks carried out. 3) Searching for, and identifying subthemes within the outcomes included in the adapted socio-ecological framework for MHM, 4) reviewing subthemes, 5) defining and naming sub-themes and 6) producing the report [51]. Codes were compared and relationships between codes were identified and analysed using analytic memos in NVivo 11.

\section{Results}

In summary, results showed that:the barriers to MHM differ depending on the person's impairment, disposable menstrual pads were preferable, but disposal practices and services were inadequate, pre and menstrual symptoms were not well understood or managed; menstrual restrictions added additional layers of challenges for people with disabilities and carers, and that there was inadequate menstrual hygiene information, training and support. 


\section{The barriers to MHM differ depending on the person's impairment}

People's experience of managing menstruation was influenced by their impairment. For instance, people with mobility impairments identified challenges related to their use of the menstrual product. Some reported that the type and positioning of the product made it uncomfortable to sit in a wheelchair all day. Many other participants with mobility impairments were concerned that the product was not absorbent enough and worried about leakages.

"When I sit in the wheelchair the pads may fold or something like that might happen which makes me feel uneasy [...]. It becomes very uncomfortable to sit. Unlike my sisters who keep moving around, I have to sit in a place continuously. I get angry then and it gets difficult"(person with multipleimpairments).

Inaccessible WASH facilities affected people with mobility impairments most severely. They were unable to easily or safely reach the place they change their menstrual product, comfortably change, wash or dry their menstrual product, or wash their bodies in private.

"II] need to wash the menstrual cloth in the toilet. There is no water in the toilet.... I have to carry water in a bucket while also managing the crutches [...] I can't wash [the menstrual] cloths either. [......] I keep it under my bed when I can't wash it, and wash it when I get water. I have a problem during menstruation when there is no water" (person with a mobility impairment).

"For those with spinal cord injury, it is easier and necessary for them to use this kind of [raised, Western] toilet. During their period they can't stand to change their pads so these kinds of toilet become more essential" (person with a mobility impairment).

All of the people with disabilities who participated in PhotoVoice took images to show how inaccessible WASH facilities presented a major challenge. Of the nine photos taken by Sharmila and Babita (who have mobility and/or self-care impairments), five images related to inaccessible WASH facilities (Figs. 1, 2, 3, 4 and 5, Additional file 4: PhotoVoice images). Tulasa, who has a self-care impairment, took four photos and one related to a lack of safe and private WASH facilities (Fig. 6, Additional file 4: PhotoVoice images).

Carers reported that some participants with intellectual impairments, had difficulties retaining MHM information. Carers repeatedly told them how to change and

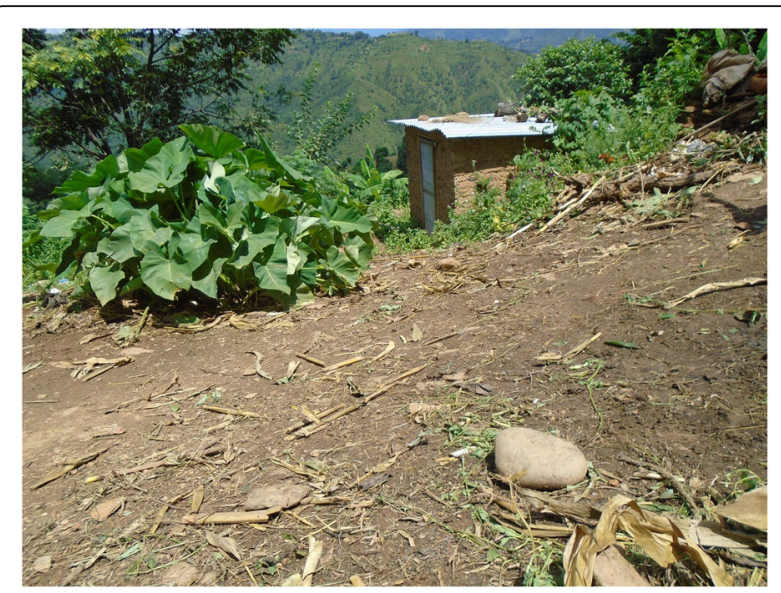

Fig. 1 "It is difficult to go to the toilet." PhotoVoice image taken by Sharmila Tamang. Ranked 1 out of 4

wash the menstrual cloth every month and every time it needed changing, but found this frustrating.

People with visual impairments highlighted difficulties seeing blood on clothes and bed sheets, and disposing of the product discretely. This was stressful and worrying because of the prevailing menstrual taboos:

"While washing the pants we know which parts to wash properly, but with the bed sheets, I cannot see the stains so it is difficult for me to clean the stains properly[....]. For throwing the pads in the dustbin, sometimes the dustbin may be outside the toilet, so I might have to throw it outside of the toilet. At those times I feel worried that a male person from my

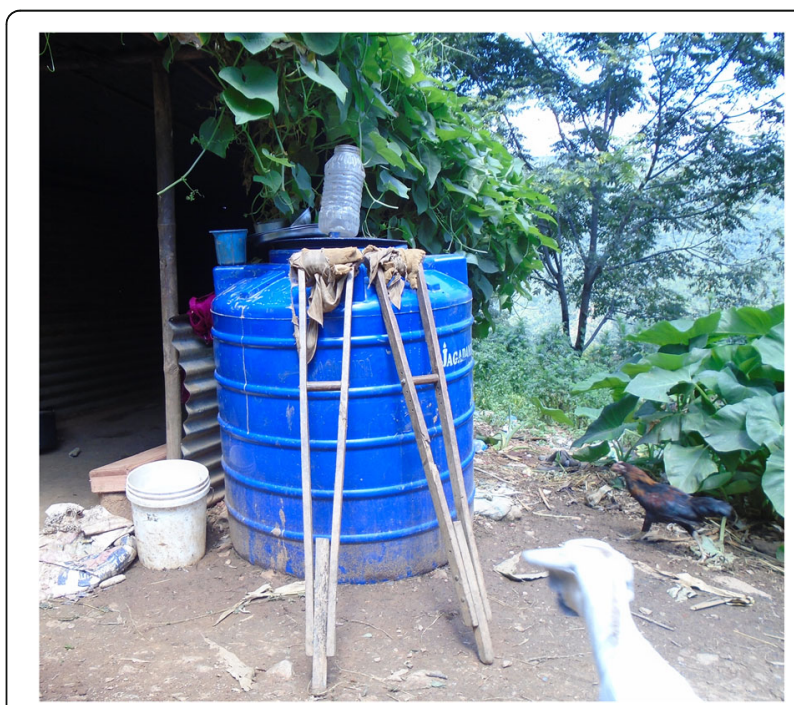

Fig. 2 "Water issue is also there, I would have to carry water which is difficult." PhotoVoice image taken by SharmilaTamang. Ranked 2 out of 4 . 


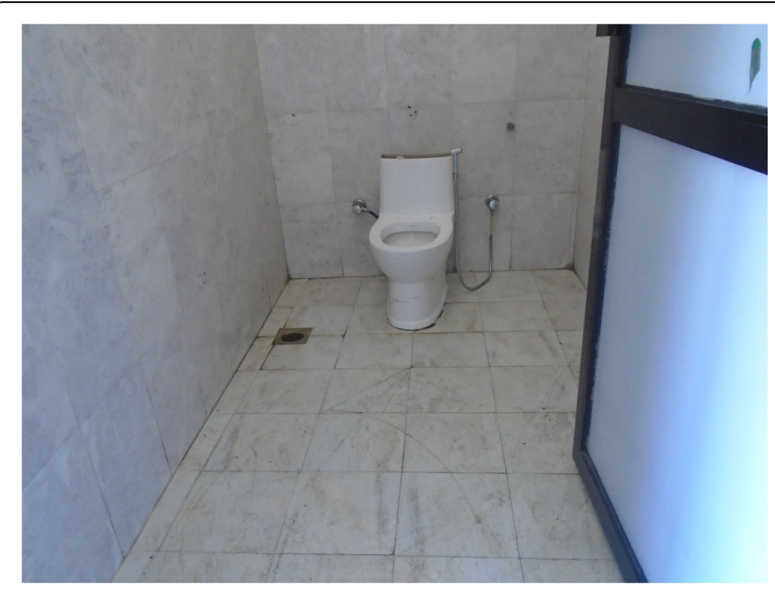

Fig. 3 "It is not only easier to use this type of toilet for those with spinal cord injury but it is a necessity. So even in villages, these types of toilets should be built for people as not everyone can live in the cities. During period also it is difficult to stand to change pads so these kind is easier to use." PhotoVoice image taken by Babita Thapa. Ranked 1 out of 5

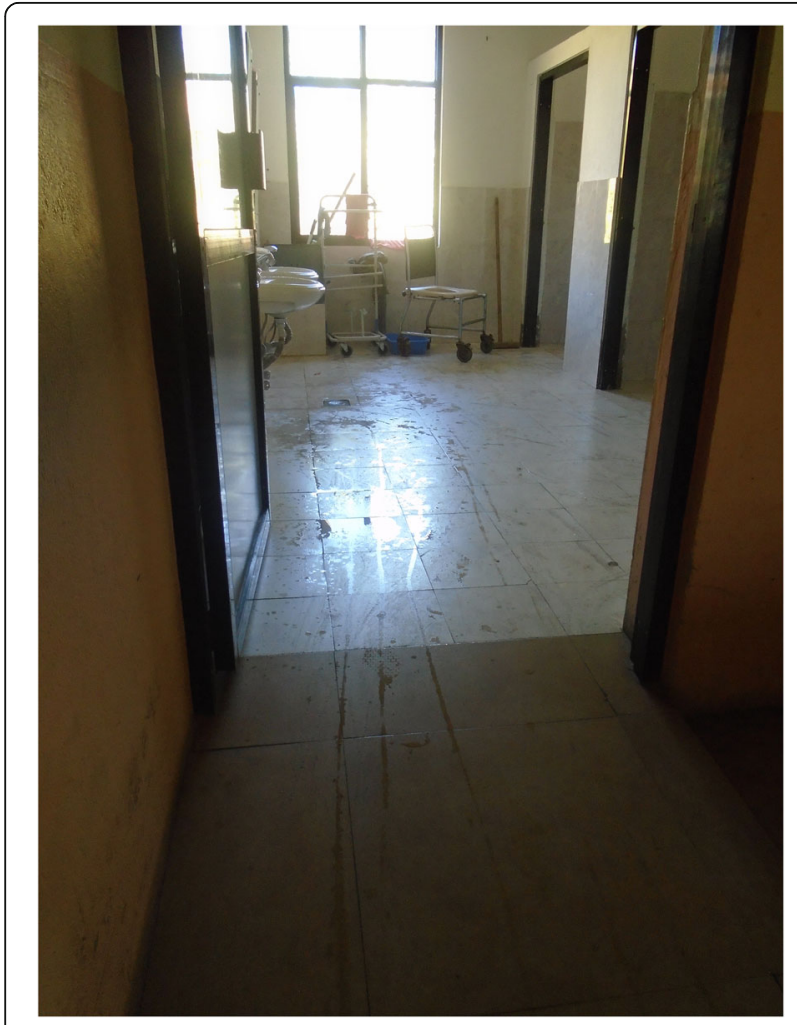

Fig. 4 "During period one should be careful using bathroom. Our legs might already feel weak so we might fall down and meet an accident. Here the toilets are made for everyone to use but if it was to built at home for crutches user, it is to be made in a way that it is not slippery. Marble should not be used as it is slippery." PhotoVoice image taken by BabitaThapa. Ranked 3 out of 5

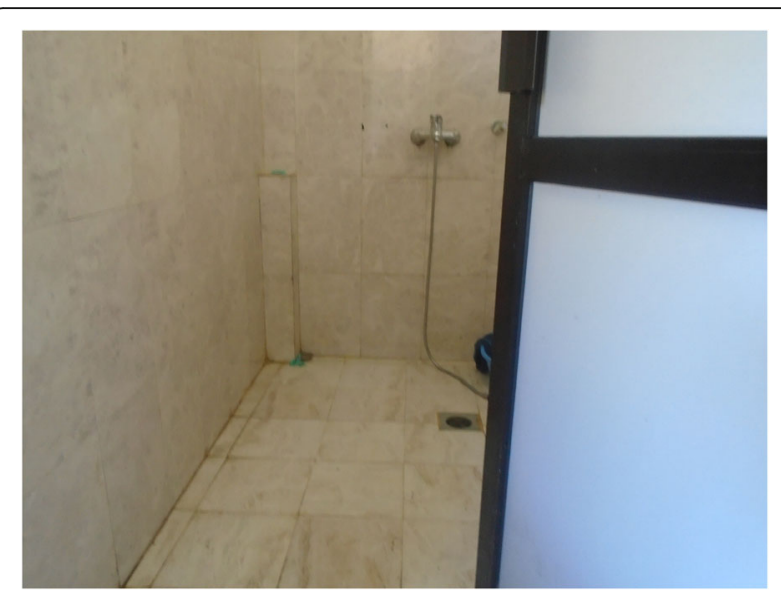

Fig. 5 "For us to be able to wash our own clothes, bathroom should be made in such a way that we can wash our clothes ourselves while sitting on wheelchair. It will be much easier. I can't wash clothes while standing or sitting. At home, I sit in a small stool but there is none here. If the washing space could be reached while sitting on a wheelchair, it would be good." PhotoVoice image taken by Babita Thapa. Ranked 5 out of 5

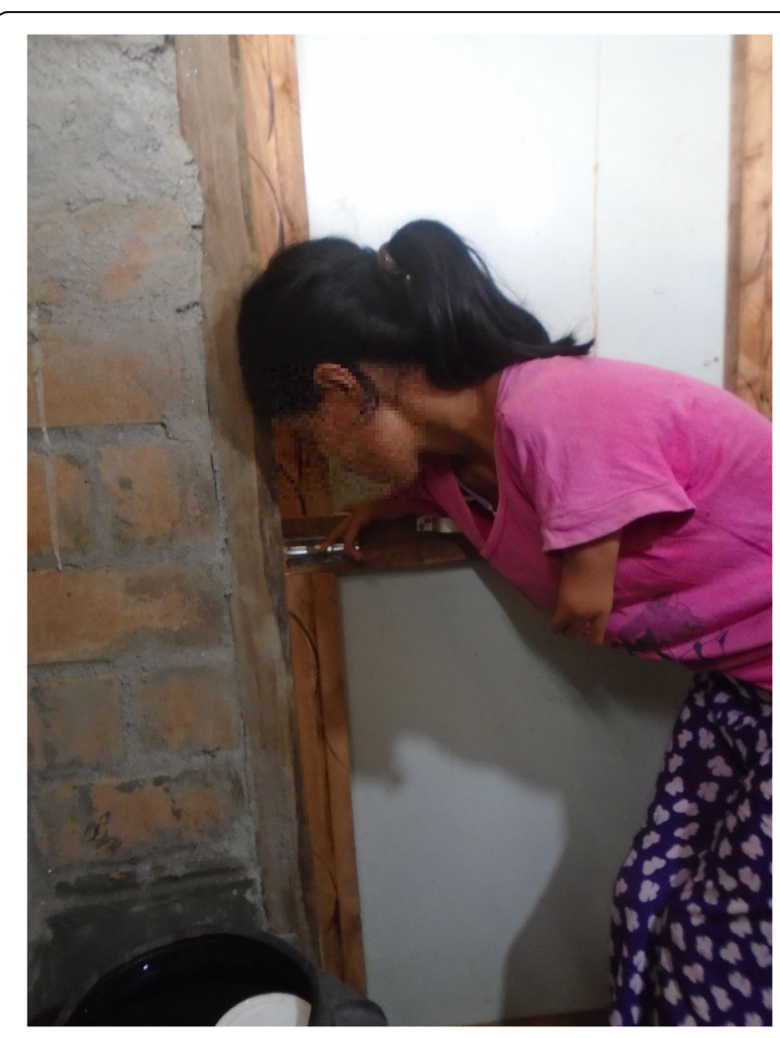

Fig. 6 "When I have to use the toilet, I need someone else to help with the latch otherwise I can't do it myself." PhotoVoice image taken by Tulasa Karki. Ranked 3 out of 4 
family would see them (person with a visual impairment).

Participants with self-care impairments, who relied on carers, felt humiliated when asking another person to change their menstrual product, and guilt seeing their carer handle their menstrual blood. Consequently, they changed their menstrual product less frequently than they wanted to.

"She says that the blood smells during my periods. [....]. She finds it disgusting. [...]. I feel bad. If I had my own hands, I wouldn't suffer so much. I wouldn't have to depend on someone else. I could do it on my own; it's not something you show it to others. I feel like crying. I feel bad" (person with self-care impairments).

"Even to change a pad I have to wait until my sister comes in the evening and helps me change, if not, I will have to wear the same pad till tomorrow" (person with self-care impairments).

In contrast, people with hearing impairments interviewed said they did not face any specific challenges explicitly related to their disability.

\section{Disposable menstrual pads are preferable, but disposal practices and services are inadequate}

All participants had access to menstrual materials, including commercial pads, menstrual cloth or tailor made pads, and results from the menstrual product market survey show that preference is highly individualised. Results of the market survey, product attribute assessment and user preference, show that disposable commercial pads with wings were preferred, and cloth was the least preferred. Table 3 captures the results across 16 people with a disability.

Though the disposable commercial pads with wings were preferred by participants, hygienic and environmentally friendly disposal behaviours were often inadequate. Many participants threw used disposable commercial pads in rivers or down hillsides, so other people were less like to see the used product. Some wrapped the pads in plastic, so they were less visible. These behaviours did not correlate to impairment type; instead, reasons include a lack of waste disposal options and little knowledge about the environmental consequences.

There were no clear preferences for product type by impairment due to the small number of participants in each category (see Tables 1 and 2 in Additional file 5: Menstrual product preference across impairment type). Through PhotoVoice, Sharmila (who has a mobility impairment) explained that she prefers using disposable commercial pads as they do not require washing, which she finds particularly challenging as she is unable to carry water and use her crutches (Fig. 2 and Fig. 7, Additional file 4: PhotoVoice images).

\section{Pre and menstrual symptoms are not well understood or managed}

Many participants said that menstrual cramps were one of the biggest challenges they face when menstruating. Pain management strategies included home remedies, such as drinking warm water, sleeping and tying a cloth tightly around the abdomen. There is a belief that commercial pain relief tablets can damage your health, so few people took them and few carers provided them.

"If I take medicine I will have more pain during the next period. That's why I don't take any medicine" (person with a mobility impairment).

Carers reported that changes in behaviour before and during menstruation for participants with intellectual impairments included withdrawal, increased hyperactivity, self-injury, showing their used menstrual product to others, excessive sleeping, being frightened, withdrawn and refusing to eat. Without social support mechanisms to help people understand and respond to the changes in behaviour, carers of people with intellectual

Table 3 Markey survey: most and least preferred menstrual product

\begin{tabular}{ll}
\hline Menstrual product & Preference \\
\cline { 2 - 3 } & Most preferred \\
\hline Disposable commercial pad with wings & 8 \\
Disposable commercial pad without wings & 2 \\
Cloth & 3 \\
Reusable tailor-made pad with wings & 3 \\
Reusable tailor-made pad without wings & 3 \\
Diaper & 0 \\
Total & 0 \\
\hline
\end{tabular}




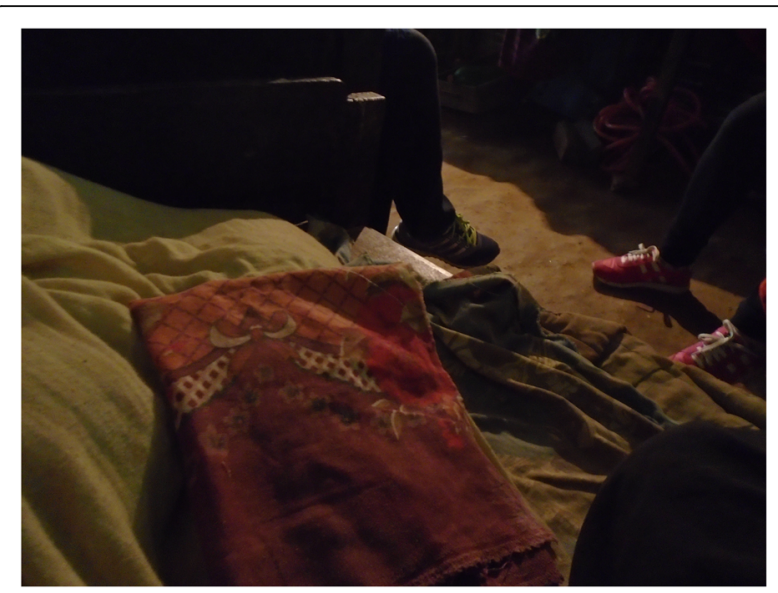

Fig. 7 "Pad is easy to use compared to cloth." PhotoVoice image taken by Sharmila Tamang. Ranked 3 out of 4

impairments felt frustrated and overwhelmed, as demonstrated by these quotes.

"When she gets her period, if I ask her if she wants to put [a menstrual] cloth [on], she would just go to her room" (carer of a person with an intellectual impairment).

"She doesn't understand, she won't listen. [....] For someone like my daughter who does understand but wouldn't remember, we can't do much" (carer of a person with an intellectual impairment).

"I feel annoyed. She doesn't listen to me" (carer of a person with an intellectual impairment).

R: "Have you ever spoken to any medical people about her menstruation?"

C: "What could we do! We can't stop it."

\section{Menstrual restrictions add additional layers of challenges} for people with disabilities and carers

Most participants followed menstrual restrictions, which dictate that menstruating people must sleep separately, are not allowed to worship, enter the kitchen, cook or touch plants, because it is believed that menstrual blood is dirty and contaminating.

"Dirty blood leaves the body during period, so we should not worship during that time" (person with a hearing impairment).

Tulasa took two photos representing the menstrual restrictions: the hut, outside her home where she sleeps when menstruating, and plants that must not be touched

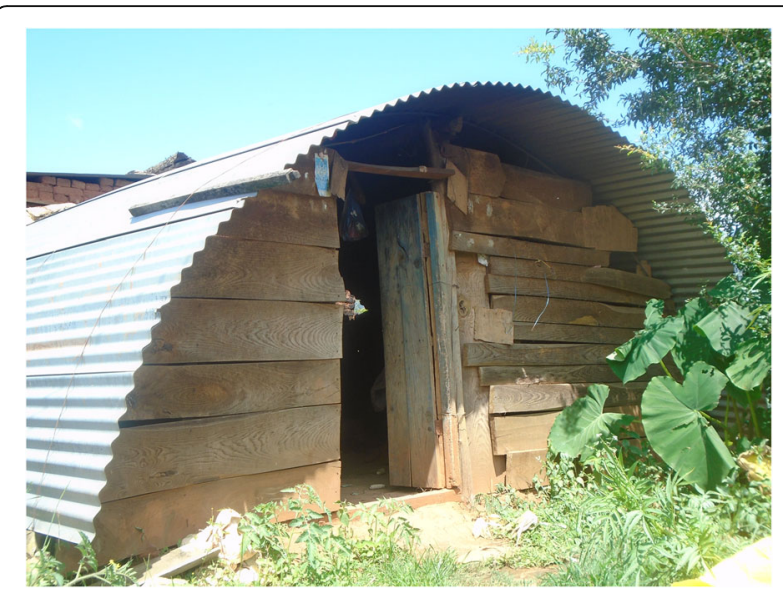

Fig. 8 "During menstruation we are not allowed to enter the house." The image is of the hut Tulasa sleeps in when menstruating. PhotoVoice image taken by Tulasa Karki. Ranked 2 out of 4

during menstruation (Figs. 8 and 9, Additional file 4: PhotoVoice images). During an in-depth interview, one participant also explained she lived in a cow shed during menstruation when at home.

"I had to be banished in the cowshed for seven to 12 days" (person with ahearing impairment).

If a person does not adhere to the restrictions, it is believed that the gods will curse the family. Disability was also viewed as a curse. Therefore, people feared that they will be doubly cursed if they did not follow restrictions.

".... am already suffering like this and people say that my disability is a curse, so if I don't obey I will be further cursed" (person with a mobility impairment).

People with visual impairments reported that menstrual restrictions were a major source of concern, fearing that they might inadvertently touch a 'restricted object', and thus lead the gods to curse the family.

"I also cannot go against my family. It has been followed by our family, it is a tradition. [...] I feel odd to move around because I am worried that I might touch [things that I should not]" (person with a visual impairment).

Two of four PhotoVoice images taken by Bishnu (a carer of a person with an intellectualimpairment), focused on menstrual restrictions (Figs. 10 and 11, Additional file 4: PhotoVoice images). She ranked these as the biggest challenges she faces when her daughter menstruates. Similarly, during in-depth interviews, carers of people 


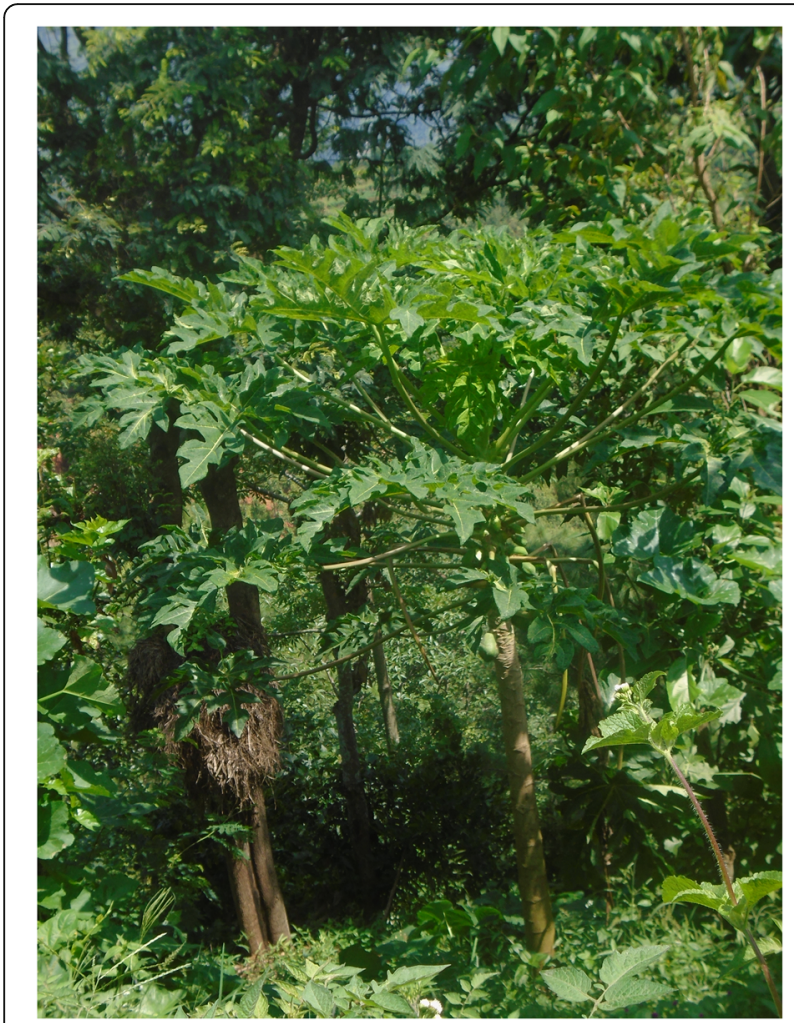

Fig. 9 "There is a belief that if you touch plants during period, it will rot." PhotoVoice image taken by Tulasa Karki. Ranked 4 out of 4

with an intellectual impairment reported being worried that their family would be cursed if restrictions were not followed. Additionally, carers of people with an intellectual impairment explained some people did not wear a menstrual cloth, preferring to soak up the menstrual blood with underwear or trousers. Some participants isolated themselves when they are menstruating and otherswent out with blood stained clothes. One carer explained:

"She just walks like that with blood on her clothes" (Carer of a person with multiple impairments).

"She would take it out and show it to others and would tell them to look at it. It was embarrassing" (Carer of a person withan intellectualimpairment).

\section{Inadequate menstrual hygiene information, training and support}

Some carers were surprised when their charges reached menarche, and one carer did not believe it when the person with a disability said they were bleeding until they saw the menstrual blood. Furthermore, information on menstrual hygiene was commonly withheld from people

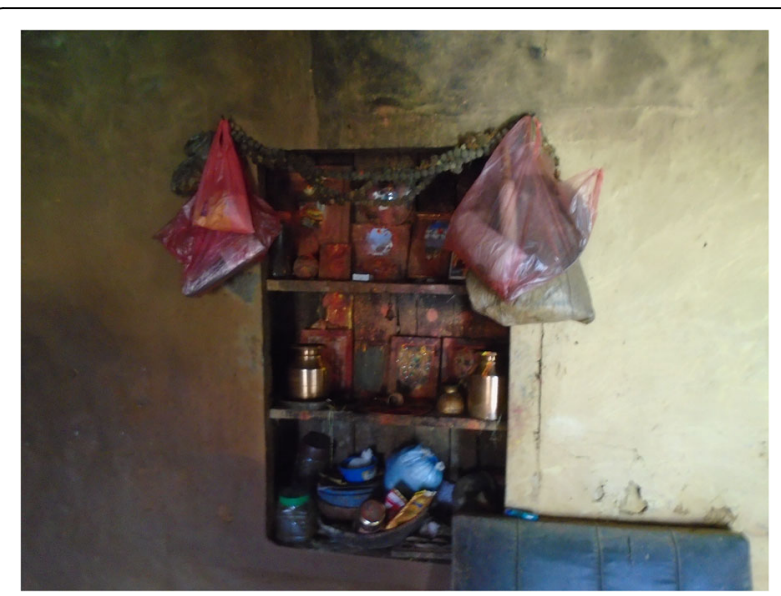

Fig. 10 "Cannot touch during period as per our tradition." The image is of worshiping area inside Bishnu's home. PhotoVoice image taken by Bishnu Maya Sapkota. Ranked 1 out of 4

with intellectual impairments, though one carer did persist and explained that her charge "took one year to understand the process and experience" (carer of a person with multiple impairments). One carer reported that a teacher sent her daughter home from school at the onset of menarche, and the carer never sent her back.

"That day, [her] teacher showed up at the house and suggested not to send [her] to the school because [she] had her mensuration in the classroom and the blood leaked on the bench she was sitting on. They said that it is difficult for [her] to take care of herself during the menstruation so it would be better that she stays at home and we take care of her. Then I stopped sending her to the school" (carer of a person with multiple impairments).

Providing menstrual care was viewed as a very private issue by carers: very few discussed this subject with other people, including medical professionals. No support or support networks existed for carers, and many carers felt isolated and overwhelmed.

"We don't know anything else. I don't go anywhere. I hear that people come to our village to teach about those things, but I haven't been taught about menstruation management" (carer of a person with multiple impairments).

Two carers of people with a self-care impairment requested MHM training for the young person in order to increase the young person's independence. A motivation for carers was fear for the future, as they worried about who would look after their daughter when they are no longer able to. 


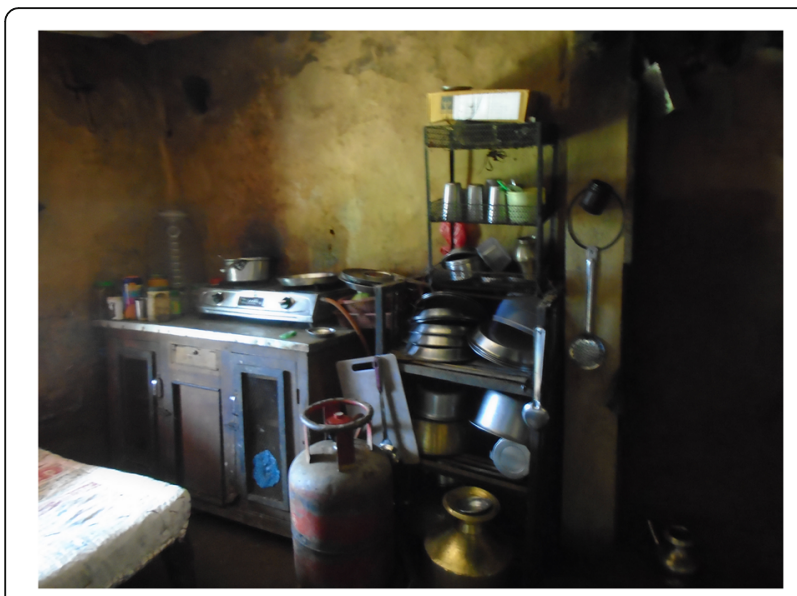

Fig. 11 "We have to follow our tradition, so should not touch kitchen during period. If one touches kitchen, worshipping area is also touched. If touched, I feel discomfort and fear that something might happen. However, in case of my daughter if she touches, god will forgive her." PhotoVoice image taken by Bishnu Maya Sapkota. Ranked 2 of 4

"For now, I am here, but in future we don't know what will be the situation. [...]. I won't live long but she has lots of time, I am very worried" (carer of a person with multiple impairments).

\section{Discussion}

This qualitative study among people with disabilities and their carers living in Nepal responds to the calls for information on the MHM barriers faced by people with disabilities, and contributes new evidence to the global discourse on MHM for the largest minority group [5, 52-55]. To the authors' knowledge, this is the only study to date which investigates the barriers experienced across all impairments. We found that the barriers to MHM are complex and differ according to the person's impairment. Furthermore, these barriers inhibit the person with a disability's ability to live a dignified life and fulfil their human rights, including going to school.

\section{The barriers to MHM differ depending on the person's impairment}

To meet the MHM requirements of people living with a disability, water, sanitation and hygiene facilities must be located close to where the person lives. For instance, people with mobility, self-care and visual impairments may require an accessible water point inside latrines, accessible locks on toilet doors, raised toilet seats, nonslippery toilet floor, accessible washing and drying area for the body, clothes and menstrual products, as well disposal mechanisms that can be easily used by everyone.
Research exploring the barriers to MHM experienced by people with different impairments and their carers in different LMIC settings must be conducted to allow for comparison. Findings should be incorporated into the global MHM agenda alongside researching MHM for school going girls and non-disabled women [56-58]. Furthermore, MHM interventions appropriate for impairment type, and carers must be developed to overcome barriers.

Participants with hearing impairments in our study explained that they did not face challenges related to their disability. However, these participants all attended a school for children with hearing impairments and said they read about menstruation in books, and were supported by friends and teachers to practically manage menstruation. This finding may not reflect the experiences of people with hearing limitations who do not attend this type of school.

The study demonstrates that people with disabilities can be separated into those who manage their menstrual cycle independently, and possibly with great difficulty, and those who are reliant on carers for MHM. MHM interventions for people without disabilities are delivered directly to the person who menstruates. Yet, for people with disabilities, there may be a third party (carer) involved, who also requires MHM information and support so that they can help another person manage their menstruation comfortably, hygienically and with dignity. Such interventions must cover all aspects of MHM, including addressing harmful social beliefs, so that people who are reliant on carers to manage their menstrual cycle do not feel humiliated when asking for support, or guilty when carers change their menstrual material. These emotions are driven by shame and disgust caused by menstrual taboos. Carers must be supported to understand that supporting another person to manage their menstrual cycle as independently as possible would benefit both parties. Additionally, low cost lifting devices to support carers bathe and change a menstrual product should also be widely promoted to support carers and protect the person with disability's dignity (see section 4.4 in Rosato-Scott et al. (2019) [59]. If carers move the person without such devices, they may experience back pain and associated issues [60].

\section{Disposable menstrual pads are preferable, but disposal practices and services are inadequate}

More research is needed to identify comfortable, appropriate and affordable menstrual products for all people with disabilities, including people who are unable to sit out of bed unaided and / or who experience incontinence. Clear information on each product option, their implications for use and disposal need to be disseminated so that people can make informed choices. Policy 
makers and implementers should be encouraged to strengthen waste management service chains and incorporate menstrual waste management within it.

\section{Pre and menstrual symptoms are not well understood or managed}

Menstrual discomfort was a key challenge expressed by participants and carers did not always manage this. A lack of pain management may have more negatively impacted people who have intellectual and / or communication impairments as they may not have understood the cause of the discomfort, or been able to communicate if they were in pain. This raises concerns that unmanaged pain may negatively impact on behaviours, which in turn can make carers feel overwhelmed, and a negative cycle forms. This is documented in three studies, which linked an inability of people on the autistic spectrum to understand the reason for menstrual discomfort or communicate when in pain. Menstrual related behaviours documented across this population in these studies included increased hyperactivity, selfinjury, fatigue and anger $[22,28,61]$.

\section{Menstrual restrictions add additional layers of challenges for people with disabilities and carers}

Menstrual taboos, including restrictions to movements, are widely followed by people with and without disabilities in Nepal $[12-14,62]$. However, due to vulnerability to violence, people with disabilities may be more susceptible to abuse when sleeping in menstrual huts [63-65]. For instance, a person with a hearing impairment may not hear an intruder approaching; people with a mobility impairment may be less able to escape, and people with communication and/or intellectual impairments may be less able to disclose experiences of abuse. Our study also highlights the fear expressed by people with disabilities that they would be 'doubly cursed' if they did not adhere to the menstrual restrictions. These findings reveal layers of stigma and discrimination faced by people with disabilities in Nepal when they menstruate.

Our findings show that carers were particularly concerned that the young person with an intellectual impairment would not follow cultural and social norms (including menstrual restrictions); that they would refuse to wear a menstrual product and would go out with menstrual blood on their clothes. In a study conducted in India, where the socio-cultural norms are similar to those of Nepal, carers reported the biggest challenge faced was that their daughters with an intellectual impairment would not wear a menstrual pad, and leave home with blood stained clothes. As a result, the carers would keep their daughters at home whilst they were menstruating, put them on long-term contraception or sterilise them [22]. When people with a disability in our study went out in public with blood stained clothes, community and family members verbally and physically abused them. Therefore, ensuring MHM for this population is also a safeguarding issue.

\section{Inadequate menstrual hygiene information, training and support}

The misconception that people with disabilities do not have the same reproductive systems as non-disabled people $[20,26]$, means they are even less likely to receive MHM information than non-disabled people. Additionally, such information was commonly withheld from people with intellectual impairments, because of the perception that they would not understand it. However, consequences of providing repetitive and simple MHM information for one participant was a greater ability to manage her own menstruation. Thus, people with intellectual impairments may be able to understand information about the menstrual cycle, if it is tailored to their level of understanding and repeated regularly. Ability to recall information would be dependent on the extent of the intellectual impairment.

MHM information is mainly delivered at schools, but many participants with intellectual impairments did not attend school so were excluded from receiving this information. One participant was sent home from school at the onset of menarche, and this marked the end of her formal education, which could negatively impact on her life chances [66]. Carers who were unable to leave the home because of caring duties were also at a disadvantage as they were unable to access MHM information shared in the community.

Our study showed that there is a lack of social support and information about how to care for another person's menstrual cycle, that menstrual care is viewed as a private issue, and that this results in carers feeling overwhelmed and isolated. These findings are reflected in studies from Taiwan, India, and the UK [22, 28, 29]. They showed that menstruation is viewed by carers (family members) as a confidential topic, so people did not speak to others or seek support from anyone, including medical professionals. This lack of support can negatively affect carer's wellbeing [5, 22, 28, 67-69].

\section{Strengths and limitations}

The strengths of this study include the use of a range of qualitative methods to explore a very private topic with people who may never have spoken about menstruation to another person. Data triangulation was applied to compare information generated through different modes, and data saturation was perceived to have been reached. Another strength was the inclusion of people 
with disabilities on the research team; we believe they were able to challenge misconceptions of carers that people with disabilities are unable to work and therefore always reliant on family members.

In terms of limitations, several possible sources of bias arose due to different types of missing data. Though participants were recruited from each impairment group (communication is included in 'multiple'), we were unable directly interview one person with an intellectual impairment. As participants in this functional domain were unable to fully understand the consent process, their carers were interviewed instead, which may not reflect their own perspectives.

The lead author's employment history includes working as a WASH practitioner focused on mainstreaming disability inclusion in development, which influenced the data collection tools applied and topics explored. For instance, experience and studies conducted dictate that people with disabilities faced more physical barriers to accessing WASH facilities than people without disabilities, so participants were observed using them and asked about barriers faced during interviews [27, 70, 71]. To minimise potential bias, a systematic review of relevant literature was conducted before the study to understand existing evidence and question personal assumptions about the barriers faced and data collection tools to apply [5].

AS and AG have a disability and we were cognisant that their experiences may introduce bias. To manage this, the week-long training for the research team included encouraging the team to mainly talk about their own experiences with participants after the interview. We also met regularly to discuss potential bias in data collection and analysis of results.

\section{Conclusion}

This study highlights the additional barriers to MHM that people with disabilities, and their carers experience, as well as the negative impacts that these have on their physical, emotional, mental and social wellbeing. Issues related to MHM for people with disabilities is even more complex than for others in the population, due to the additional disability discrimination and constraints experienced, so require innovative and adapted solutions to existing MHM approaches that often fail to reach them. Even though MHM is not explicitly included in the Sustainable Development Goals (SDGs), it is essential for achieving the goals on gender, health and education [72]. Disability is the largest minority group, so attention to, and resourcing for disability inclusive MHM must be prioritised for progress to be made within the last nine years of the SDGs, which aims to 'Leave No One Behind'.

\section{Abbreviations}

CIUD: Centre for Integrated Urban Development; CRPD: Convention on the Rights of Persons with Disabilities; KIRDAC: Karnali Integrated Rural Development and Research Centre; LMICs: Low- and middle-income countries; LSHTM: London School of Hygiene and Tropical Medicine; MHM: Menstrual hygiene management; WASH: Water, sanitation and hygiene; WEDC: Water and Engineering Development Centre

\section{Supplementary Information}

The online version contains supplementary material available at https://doi. org/10.1186/s12889-021-10439-y.

Additional file 1. In depth interview question guide, product attribute assessment (market survey) and accessibility and safety audit for carers.

Additional file 2. In depth interview question guide, product attribute assessment (market survey) and accessibility and safety audit for people with disabilities.

Additional file 3. PhotoVoice - guidance for researchers, which explains the purpose of PhotoVoice, how to identify participants, consent process, and a step by step guide on how to conduct PhotoVoice and ranking with participants.

Additional file 4. PhotoVoice images taken by participants (Figs. 1, 2, 3, $4,5,6,7,8,9,10$ and 11), ranked according to perceived level of importance. The images relate to the following results: 'The barriers to MHM differ depending on the person's impairment', 'Disposable menstrual pads are preferable, but disposable practice and service are inadequate', and 'Impacts of menstrual restrictions'.

Additional file 5. Menstrual product preference across impairment type, containing Table 1: Most preferred product according to the person's impairment, and Table 2. Least preferred product according to the person's impairment.

\section{Acknowledgements}

The authors wish to thank all research participants for their involvement in the study; WaterAid Nepal, KIRDAC, CIUD, Sian White and Robert Dreibelbis for their time, advice and support.

\section{Availability of data and material}

The authors confirm that the data supporting the findings of this study are available within the article [and/or] its supplementary materials.

\section{Authors' contributions}

Conceptualisation, JW; methodology, JW, HK, BT, and SH; training of research team, JW; formal analysis, JW; investigation, JW, SK, AS and AG; data curation, JW; supervision, JW, HK, TM, SH and BT; project management, coordination and administration, JW; funding acquisition, TM; writing — original draft preparation, JW; writing, review and editing, JW; visualisation, JW; manuscript review, HK, BT, SH, SK. All authors have read and approved the manuscript.

\section{Funding}

Funding was provided by the Bill and Melinda Gates Foundation. The funders had no role in the design of the study, data extraction, analysis, interpretation or writing of the report.

\section{Declarations}

Ethics approval and consent to participate

Ethical approval for the study was granted by from the Research Ethics Committee at the LSHTM (reference: 12091) and the Nepal Health Research Council (reference: 102/2017).

Informed consent was obtained and witnessed from each participant before enrolment. An information sheet/consent form (in Nepali) was given to, or read out to the participants by the research team. Informed written (or a thumb print if illiterate) consent was received from carers and implementers. Assent was sought from participants under 18 years, and consent sought from their carer or parent. Participants were given the option to have their carer present during the interview. Assent also was sought from people who could not fully understand the consent process, and then consent sought 
from carers, who were interviewed instead of the person with a disability. It was made clear that participation was voluntary. Methods were adapted to be fully inclusive. For example, for individuals who speak in sign language, sign language interpreters would be available. Simplified information sheets were available for participants with intellectual and communication impairments.

The ethics and consent process of PhotoVoice was very thorough to ensure that the participants understood the purpose of the activity and what they are agreeing to. Written consent was sought at the start of the process. The researcher explained how participants could take photos without showing their face and how to represent menstrual issues in a dignified way. Secondary written consent was sought after the photos were taken, the participant viewed the photos, the interview conducted and the photo ranking so the participant could make a better judgement about how they wish them to be used. This consent related to how the photos could be used and if the photographer wanted to be credited by their real name or a pseudonym. All participants requested that their real names be credited whenever their photos and captions are used.

\section{Consent for publication}

Participants gave explicit written consent for their identifying images to be published in this study. Participants own their images taken during PhotoVoice and requested that their real names are credited when their photos are used. No individual or identifiable personal details gained through in-depth interviews are included in this manuscript.

\section{Competing interests}

The authors declare that they have no competing interests.

\section{Author details}

${ }^{1}$ London School of Hygiene \& Tropical Medicine, Keppel Street, London WC1E 7HT, UK. ${ }^{2}$ Independent consultant, Kathmandu, Nepal. ${ }^{3}$ WaterAid, 27-29 Durham Street, London SE11 5JD, UK.

\section{Received: 28 April 2020 Accepted: 15 February 2021}

\section{Published online: 10 March 2021}

\section{References}

1. Sommer M, Caruso BA, Sahin M, Calderon T, Cavill S, Mahon T, et al. A time for global action: addressing girls' menstrual hygiene management needs in schools. PLoS Med. 2016;13(2):e1001962.

2. Hennegan J, Shannon A, Rubli J, Schwab K, Melendez-Torres G. Women's and girls' experiences of menstruation in low- and middle-income countries: a systematic review and qualitative metasynthesis. PLoS Med. 2019;16:e1002803.

3. Winkler I, Roaf V. Taking the bloody linen out of the closet: menstrual hygiene as a priority for achieving gender equality. Cardozo Journal of Law and Gender. 2015

4. Charlifue SW, Gerhart KA, Menter RR, Whiteneck GG, Manley MS. Sexual issues of women with spinal cord injuries. Paraplegia. 1992;30(3):192-9.

5. Wilbur J, Torondel B, Hameed S, Mahon T, Kuper H. Systematic review of menstrual hygiene management requirements, its barriers and strategies for disabled people. PLoS One. 2019;14(2):e0210974.

6. Wilson E, Haver J, Torondel B, Rubli J, Caruso BA. Dismantling menstrual taboos to overcome gender inequality. The Lancet Child \& Adolescent Health. 2018;2(8):e17.

7. Neumeyer $\mathrm{H}$, Klasing A. Understanding Menstrual Hygiene Management and human rights. Menstrual Hygiene Day; 2016.

8. Hennegan J, Torondel B, Phillips-Howard PA, Sommer M, Montgomery P. Time to talk about menstruation: a response. Lancet. 2017;390(10097):845-6.

9. PSI Nepal. 2017 Scoping Review and Preliminary Mapping Menstrual Health and Hygiene Management in Nepal 2017.

10. WaterAid. Is menstrual hygiene and management an issue for adolescent school girls? A comparative study of four schools in different settings in Nepal. 2009

11. Crawford M, Menger L, Kaufman M. 'This is a natural process': managing menstrual stigma in Nepal. Culture. 2014;16.

12. Karki KB, Poudel, P. C., Rothchild, J., Pope, N., Bobin, N. C., Gurung, Y., Basnet, M., Poudel, M.., Sherpa LY. Scoping Review and Preliminary Mapping: Menstrual Health and Hygiene Management in Nepal. 2017.
13. Amatya P, Ghimire S, Callahan KE, Baral BK, Poudel KC. Practice and lived experience of menstrual exiles (Chhaupadi) among adolescent girls in farwestern Nepal. PLoS One. 2018;13(12):e0208260.

14. Budhathoki SS, Bhattachan M, Castro-Sanchez E, Sagtani RA, Rayamajhi RB, Rai $P$, et al. Menstrual hygiene management among women and adolescent girls in the aftermath of the earthquake in Nepal. BMC Womens Health. 2018;18(1):33.

15. Government of Nepal CBoS. National Population and Housing Census 2011 (National Report). 2012.

16. Baumann SE, Lhaki P, Burke JG. Assessing the role of caste/ethnicity in predicting menstrual knowledge, attitudes, and practices in Nepal. Glob Public Health. 2019;14(9):1288-301.

17. Ranabhat C, Kim C-B, Choi EH, Aryal A, Park MB, Doh YA. Chhaupadi culture and reproductive health of women in Nepal. Asia Pacific Journal of Public Health. 2015;27(7):785-95.

18. Hennegan J, Brooks DJ, Schwab KJ, Melendez-Torres GJ. Measurement in the study of menstrual health and hygiene: A systematic review and audit. PLoS ONE. 2020;15 (6) (no pagination)(e0232935).

19. Sommer M, Schmitt ML, Clatworthy D, Bramucci G, Wheeler E, Ratnayake R. What is the scope for addressing menstrual hygiene management in complex humanitarian emergencies? A global review. Waterlines. 2016;35(3): 245-64.

20. WHO, World Bank. World report on disability. 2011

21. Dündar T, Özsoy S. Menstrual hygiene management among visually impaired women. British Journal of Visual Impairment. 2020.

22. Thapa P, Sivakami M. Lost in transition: menstrual experiences of intellectually disabled school-going adolescents in Delhi, India. Waterlines. 2017;36(4):317-38.

23. WSSCC. Leave no one behind: an MHM toolkit for women and girls with visual and hearing impairments. 2017.

24. Wilbur J, Bright T, Mahon T, Hameed S, Torondel B, Mulwafu W, et al. Developing behaviour change interventions for improving access to health and hygiene for people with disabilities: two case studies from Nepal and Malawi. Int J Environ Res Public Health. 2018;15(12):2746.

25. House S, Mahon T, Cavill S. Menstrual hygiene matters; a resource for improving menstrual hygiene around the world. 2012.

26. WHO, UNFPA. Promoting sexual and reproductive health for persons with disabilities WHO/UNFPA guidance note. 2009.

27. Wilbur J, Jones H, Gosling L, Groce N, Challenger E. Undoing inequity: inclusive water, sanitation and hygiene programmes that deliver for all in Uganda and Zambia. 336th WEDC international conference; Nakuru, Kenya: WEDC; 2013.

28. Chou YC, Lu ZY. Caring for a daughter with intellectual disabilities in managing menstruation: a mother's perspective. J Intellect Develop Disabil. 2012;37(1):1-10.

29. Van der Merwe JV, Roux JP. Sterilization of mentally retarded persons. Obstetrical \& Gynecological Survey. 1987;42(8):489-93.

30. Perrin JC, Sands CR, Tinker DE, Dominguez BC, Dingle JT, Thomas MJ. A considered approach to sterilization of mentally retarded youth. Am J Dis Child. 1976;130(3):288-90.

31. Kirkham YA, Allen L, Kives S, Caccia N, Spitzer RF, Ornstein MP. Trends in menstrual concerns and suppression in adolescents with developmental disabilities. J Adolesc Health. 2013;53(3):407-12.

32. van Schrojenstein Lantman-deValk HMJ, Rook F, Maaskant MA. The use of contraception by women with intellectual disabilities. J Intellect Disabil Res. 2011;55:434-40.

33. Hennegan J. Menstrual Hygiene Management and Human Rights: The Case for an Evidence-Based Approach, Women's Reproductive Health. Women's Reproductive Health. 2017;4(3).

34. United W, Watch HR. Understanding Menstrual Hygiene Management \& Human Rights 2017

35. Creswell JW. Qualitative inquiry and research design: choosing among five approaches. Second edition ed: Sage Publications; 2007.

36. Durham J, Brolan CE, Mukandi B. The convention on the rights of persons with disabilities: a foundation for ethical disability and health research in developing countries. Am J Public Health. 2014;104(11):2037-43.

37. Bhatta C, Bhujel S, Dhakal S, Gyawali N, Shah MK, Shrestha S, et al. Strengthening the voices of adolescents with disabilities in Nepal. International Centre for Evidence in Disability: LSHTM; 2018.

38. Causemann B, Gohl E. Guide to lifeline, a tiny tool (v1.3). NGO-IDEAs; 2012. 
39. London School of Hygiene and Tropical Medicine (LSHTM), International Centre for Evidence in Disability (ICED), CBM, CSF, Hambisela. Getting to know Cerebral Palsy. Working with parent groups - a training resource for facilitators, parents, caregivers, and persons with cerebral palsy.

40. Mahon T, Cavill S. Menstrual Hygiene Matters: Training Guide for Practitioners. WaterAid; 2015.

41. Gerstenblatt P. Collage portraits as a method of analysis in qualitative research. Int J Qual Methods. 2013;12(1):294-309.

42. Zuurmond $M$, Kayestha $S$, Lamsal $P$, Panthee $M$, Wilbur J. Research training workshop - Nepal (28 August - 1 September, 2017): Building the capacity of youth researchers for young people living with a disability.: International Centre for Evidence in Disablity (ICED); 2017.

43. Government of Nepal CBoS. Nepal Living Standards Survey 2010/11 2011 [Available from: https://microdata.worldbank.org/index.php/catalog/1000].

44. Washington Group on Disability Statistics. Recommended short set of questions. 2010.

45. Kruger D. An introduction to phenomenological psychology: Juta academic; 1988.

46. Patton MQ. Qualitative Research \& Evaluation Methods: thousand oaks. California: Sage Publications; 2002.

47. Browne K. Snowball sampling: using social networks to research nonheterosexual women. Int J Soc Res Methodol. 2005;8(1):47-60.

48. Palinkas LA, Horwitz SM, Green CA, Wisdom JP, Duan N, Hoagwood K. Purposeful sampling for qualitative data collection and analysis in mixed method implementation research. Admin Pol Ment Health. 2015;42(5):533-44.

49. WEDC, WaterAid. Accessibility and safety audit: latrine. 2013.

50. Patton MQ. Enhancing the quality and credibility of qualitative analysis. Health Serv Res. 1999;34(5 Pt 2):1189-208.

51. Braun V, Clarke V. Using thematic analysis in psychology. Qual Res Psychol. 2006;3(2):77-101.

52. Phillips-Howard PA, Caruso B, Torondel B, Zulaika G, Sahin M, Sommer M. Menstrual hygiene management among adolescent schoolgirls in low- and middle-income countries: research priorities. Glob Health Action. 2016;9: 33032.

53. Republic of South Africa DW, 2018 MHMS, UNFPA. First East and Southern Africa Regional Symposium Improving Menstrual Health Management for Adolescent Girls and Women. 2018

54. UNICEF. Guidance on Menstrual Health and Hygiene. 2019.

55. Columbia University, UNICEF. MHM in Ten: Advancing the MHM agenda in Schools. 2016

56. Daniels GJ. Investigating fear, Shyness, And Discomfort Related To Menstrual Hygiene Management In Rural Cambodia: Yale; 2016.

57. Sommer M, Phillips-Howard PA, Mahon T, Zients S, Jones M, Caruso BA. Beyond menstrual hygiene: addressing vaginal bleeding throughout the life course in low and middle-income countries. BMJ Global Health. 2017;2(2).

58. Carrard N, Neumeyer H, Pati BK, Siddique S, Choden T, Abraham T, et al. Designing human rights for duty bearers: making the human rights to water and sanitation part of everyday practice at the local government level. Water. 2020;12

59. Rosato-Scott C, Giles-Hansen C, House S, Wilbur J, Macaulay M, Barrington D, et al. Guidance on supporting people with incontinence in humanitarian and low- and middle-income contexts (LMICs). 2019.

60. Ansari Z. Understanding the coping mechanisms employed by people with disabilities and their families to manage incontinence in Pakistan. London: London School of Hygiene and Tropical Medicine; 2017.

61. Kyrkou M. Health issues and quality of life in women with intellectual disability. J Intellect Disabil Res. 2005;49(10):770-2.

62. WaterAid. Menstrual hygiene management in Udaypur and Sindhuli districts of Nepal 2016.

63. Dammeyer J, Chapman M. A national survey on violence and discrimination among people with disabilities. BMC Public Health. 2018;18(1):355.

64. Basile KC, Breiding MJ, Smith SG. Disability and risk of recent sexual violence in the United States. Am J Public Health. 2016;106(5):928-33.

65. Hughes K, Bellis MA, Jones L, Wood S, Bates G, Eckley L, et al. Prevalence and risk of violence against adults with disabilities: a systematic review and meta-analysis of observational studies. Lancet. 2012;379(9826):1621-9.

66. United Nations. Quality Education: why it matters.

67. Carnaby S, Cambridge P. Getting personal: an exploratory study of intimate and personal care provision for people with profound and multiple intellectual disabilities. J Intellect Disabil Res. 2002;46(Pt 2):120-32.
68. Lin LP, Lin PY, Chu CM, Lin JD. Predictors of caregiver supportive behaviors towards reproductive health care for women with intellectual disabilities. Res Dev Disabil. 2011;32(2):824-9.

69. Lin LP, Lin JD, Chu C, Chen LM. Caregiver attitudes to gynaecological health of women with intellectual disability. J Intellect Develop Disabil. 2011;36(3): 149-55.

70. Wilbur J, Jones H. Disability: making CLTS fully inclusive. CLTS Knowledge Hub: Institute of Development Studies; 2014.

71. Desai G, Wilbur J, Smith K, Jensen JN, Lenker J, Ram P. Laddering up? A research framework in sanitation for people with disabilities in low-income settings Watelines. 2017.

72. SIMAVI, PATH, United W. MHM and the SDGs. Menstrual Hygiene Management Matters to the achievement of several Sustainable Development Goals (SDGs) 2020.

\section{Publisher's Note}

Springer Nature remains neutral with regard to jurisdictional claims in published maps and institutional affiliations.
Ready to submit your research? Choose BMC and benefit from:

- fast, convenient online submission

- thorough peer review by experienced researchers in your field

- rapid publication on acceptance

- support for research data, including large and complex data types

- gold Open Access which fosters wider collaboration and increased citations

- maximum visibility for your research: over $100 \mathrm{M}$ website views per year

At BMC, research is always in progress.

Learn more biomedcentral.com/submissions 